\title{
FEEDBACK INCREASES NEITHER THE FADING NUMBER NOR THE PRE-LOG
}

\author{
Amos Lapidoth $\quad$ Stefan M. Moser \\ Signal and Information Processing Laboratory \\ Department of Information Technology and Electrical Engineering \\ Swiss Federal Institute of Technology (ETH) Zurich \\ CH-8092 Zurich, Switzerland \\ e-mail: $\{$ lapidoth, moser\}@isi.ee.ethz.ch
}

\begin{abstract}
The feedback capacity of a single-input single-output fading channel with memory is investigated. It is shown that asymptotically, as the signal-to-noise ratio (SNR) tends to infinity, noiseless feedback does not increase capacity.

More specifically, for finite-energy stationary and ergodic fading processes that are regular (i.e., that have a finite differential entropy rate) feedback does not increase the fading number; and for Gaussian fading processes that are non-regular feedback does not increase the pre-log.
\end{abstract}

Index Terms - Channel Capacity, Fading Channels, Fading Number, Feedback, High SNR, Pre-Log, Regular and Non-Regular Fading Processes.

\section{INTRODUCTION}

In [1] the capacity of fading channels with memory was investigated. It was shown there that for any finite-energy regular fading process $\left\{H_{k}\right\}$ (i.e., one having a finite entropy rate) the channel capacity $C$ grows asymptotically only double-logarithmically in the signal-to-noise ratio (SNR). The fading number $\chi$ was introduced as the second term in the high-SNR expansion of the capacity

$$
\chi\left(\left\{H_{k}\right\}\right) \triangleq \lim _{\mathrm{SNR} \uparrow \infty}\{C(\mathrm{SNR})-\log \log \mathrm{SNR}\},
$$

where $C$ stands for the capacity with peak- or average-power constraint, and its value was computed.

Subsequently [2], [3], [4], for the more restricted Gaussian fading and for peak-power constraints, the analysis of non-regular fading processes (i.e., processes of differential entropy rate negative infinity) was completed. It was shown that while for regular fading processes the channel capacity grows like $\log \log \mathrm{SNR}$, this is not necessarily the case for non-regular fading. Depending on the spectrum $\mathrm{F}(\cdot)$ of

The work of S. M. Moser was supported in part by the ETH under TH-23 02-2. the non-regular Gaussian fading process, the asymptotic behavior of the channel capacity can be varied, e.g., doublelogarithmic, logarithmic, or a fractional power thereof. The pre-log $\Pi^{\mathrm{PP}}$ was defined as

$$
\Pi^{\mathrm{PP}} \triangleq \lim _{\mathrm{SNR} \uparrow \infty} \frac{C^{\mathrm{PP}}(\mathrm{SNR})}{\log \mathrm{SNR}},
$$

where $C^{\mathrm{PP}}$ denotes capacity with a peak-power constraint, and its value was computed, see (19).

In this paper we extend these results to the case where there is a noiseless feedback link from the receiver to the transmitter. We show that for fading channels with memory feedback does not increase the fading number or the pre-log.

\section{CHANNEL MODEL}

We consider a communication system as depicted in Fig. 1. A message $M$ is transmitted over a single-input single-out-

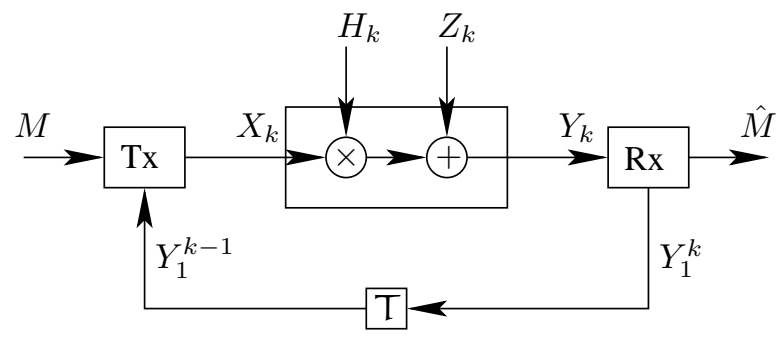

Fig. 1. The communication system under consideration.

put fading channel with memory. The channel output at time $k$ is given by

$$
Y_{k}=H_{k} x_{k}+Z_{k}
$$

where $x_{k} \in \mathbb{C}$ denotes the time- $k$ channel input; the random variable $H_{k} \in \mathbb{C}$ denotes the time- $k$ fading term; and $Z_{k} \in$ $\mathbb{C}$ denotes the time- $k$ additive noise term. 
The additive noise process $\left\{Z_{k}\right\}$ is assumed to be a white zero-mean circularly-symmetric complex Gaussian process, i.e.,

$$
\left\{Z_{k}\right\} \sim \operatorname{IID} \mathcal{N}_{\mathbb{C}}\left(0, \sigma^{2}\right)
$$

for some $\sigma^{2}>0$.

Neither transmitter nor receiver know the realization of the fading process $\left\{H_{k}\right\}$; they only know its law. We consider two different scenarios. In the first scenario $\left\{H_{k}\right\}$ is stationary, ergodic, of finite energy

$$
\mathrm{E}\left[\left|H_{k}\right|^{2}\right]<\infty,
$$

and of finite differential entropy rate

$$
h\left(\left\{H_{k}\right\}\right)>-\infty .
$$

In the second scenario we address the case where the entropy rate $h\left(\left\{H_{k}\right\}\right)$ need not be finite, but we restrict ourselves to Gaussian fading. I.e., we assume that $\left\{\tilde{H}_{k}\right\} \triangleq$ $\left\{H_{k}-d\right\}$ is a zero-mean, finite-variance, stationary circularly-symmetric Gaussian process of arbitrary spectral distribution function $\mathrm{F}(\lambda),-1 / 2 \leq \lambda \leq 1 / 2$, where $d \in \mathbb{C}$ denotes the spectral component of the process.

We always assume that $\left\{Z_{k}\right\}$ and $\left\{H_{k}\right\}$ are independent and that their joint law does not depend on the channel input.

Finally, we allow noiseless feedback from the receiver to the transmitter. The feedback is delayed by one time-step, so that the feedback random vector available to the transmitter at time instant $k$ consists of all past channel outputs $Y_{1}^{k-1}$.

We consider two types of power constraints: an averagepower constraint and a peak-power constraint. Under the former we require that for any message $m \in\left\{1, \ldots,\left\lfloor e^{n \mathbb{R}}\right\rfloor\right\}$

$$
\frac{1}{n} \sum_{k=1}^{n} \mathrm{E}\left[\left|X_{k}\left(m, Y_{1}^{k-1}\right)\right|^{2}\right] \leq \mathcal{E}_{\mathrm{s}},
$$

were $X_{k}\left(m, Y_{1}^{k-1}\right)$ denotes the symbol transmitted at time $k$ to convey message $m$ after the symbols

$$
Y_{1}^{k-1}=\left(Y_{1}, \ldots, Y_{k-1}\right)
$$

were received; $n$ denotes the blocklength; and $\mathbb{R}$ denotes the rate in nats per channel use.

Under the peak-power constraint we replace (7) with the almost sure constraint

$$
\left|X_{k}\left(m, Y_{1}^{k-1}\right)\right|^{2} \leq \mathcal{E}_{\mathrm{s}}, \quad 1 \leq k \leq n .
$$

The signal-to-noise ratio (SNR) is defined in both situations by

$$
\mathrm{SNR} \triangleq \frac{\mathcal{E}_{\mathrm{s}}}{\sigma^{2}}
$$

The subject of our investigation is the capacity per channel use which we denote by $C$ (SNR). To clarify notation we will use superscripts "Avg" and "PP" to denote the capacity under average-power and peak-power constraints, respectively, and a subscript "FB" whenever feedback is available. Clearly,

$$
C^{\mathrm{PP}}(\mathrm{SNR}) \leq C^{\mathrm{Avg}}(\mathrm{SNR}) \leq C_{\mathrm{FB}}^{\mathrm{Avg}}(\mathrm{SNR}),
$$

and

$$
C^{\mathrm{PP}}(\mathrm{SNR}) \leq C_{\mathrm{FB}}^{\mathrm{PP}}(\mathrm{SNR}) .
$$

Consequently, for the corresponding fading numbers

$$
\begin{gathered}
\chi^{\mathrm{PP}}\left(\left\{H_{k}\right\}\right) \leq \chi^{\mathrm{Avg}}\left(\left\{H_{k}\right\}\right) \leq \chi_{\mathrm{FB}}^{\mathrm{Avg}}\left(\left\{H_{k}\right\}\right), \\
\chi^{\mathrm{PP}}\left(\left\{H_{k}\right\}\right) \leq \chi_{\mathrm{FB}}^{\mathrm{PP}}\left(\left\{H_{k}\right\}\right),
\end{gathered}
$$

and for the corresponding pre-logs

$$
\Pi^{\mathrm{PP}} \leq \Pi_{\mathrm{FB}}^{\mathrm{PP}} \text {. }
$$

\section{REGULAR FADING}

In [1] it is shown that for regular fading the fading number $\chi$ as defined in (1) is given by

$$
\begin{aligned}
\chi^{\mathrm{PP}}\left(\left\{H_{k}\right\}\right) & =\chi^{\mathrm{Avg}}\left(\left\{H_{k}\right\}\right) \\
& =\log \pi+\mathrm{E}\left[\log \left|H_{1}\right|^{2}\right]-h\left(\left\{H_{k}\right\}\right),
\end{aligned}
$$

where

$$
h\left(\left\{H_{k}\right\}\right) \triangleq \lim _{n \rightarrow \infty} \frac{1}{n} h\left(H_{1}^{n}\right) .
$$

We now extend these results to the case where there is noiseless feedback from the receiver to the transmitter.

Theorem 1. Let a fading channel be defined as in Section 2 with a fading process that is stationary, ergodic, of finite energy, and of finite differential entropy rate (6). Then noiseless feedback from the receiver to the transmitter does not increase the asymptotic channel capacity, i.e.,

$$
\lim _{\operatorname{SNR} \uparrow \infty}\left\{C_{\mathrm{FB}}(\mathrm{SNR})-C(\mathrm{SNR})\right\}=0,
$$

where the above holds irrespective of weather an averagepower constraint (7) or a peak-power constraint (8) is imposed.

That is, the fading number in the presence of noiseless feedback $\chi_{\mathrm{FB}}\left(\left\{H_{k}\right\}\right)$ is given by (15).

The intuition for this result is that feedback only helps in improving the input power allocation and that this improvement is negligible in the log log-regime.

Remark 2. Theorem 1 can be extended to the case where the receiver has access to some partial side-information $\left\{\mathbf{S}_{k}\right\}$ about the fading process. It is assumed that the fading process $\left\{H_{k}\right\}$ and the receiver side-information $\left\{\mathbf{S}_{k}\right\}$ 
are jointly stationary, ergodic, and of finite mutual information rate $I\left(\left\{H_{k}\right\} ;\left\{\mathbf{S}_{k}\right\}\right)$,

$$
\lim _{n \rightarrow \infty} \frac{1}{n} I\left(H_{1}^{n} ; \mathbf{S}_{1}^{n}\right)<\infty .
$$

Moreover, $\left(\left\{H_{k}\right\},\left\{\mathbf{S}_{k}\right\}\right)$ are independent of the additive noise $\left\{Z_{k}\right\}$, and the joint law of $\left(\left\{H_{k}\right\},\left\{\mathbf{S}_{k}\right\},\left\{Z_{k}\right\}\right)$ does not depend on the channel input; see [1].

Then feedback (which now consists of all past channel outputs and all past side-information variables) does not increase the fading number $\chi\left(\left\{H_{k}\right\} \mid\left\{\mathbf{S}_{k}\right\}\right)$.

As a matter of fact, the fading number is not increased even if the side-information is revealed to the transmitter in an acausal fashion, i.e., the transmitter learns the realization of $\mathbf{S}_{1}^{n}$ before the transmission begins.

\section{NON-REGULAR GAUSSIAN FADING}

In [2], [3], it was shown that the pre-log $\Pi^{\mathrm{PP}}$ as defined in (2) is determined by the ratio of the total length of the frequency bands where the spectral density is null to the total frequencies:

$$
\Pi^{\mathrm{PP}}=\mu\left(\left\{\lambda: \mathrm{F}^{\prime}(\lambda)=0\right\}\right),
$$

where $\mu(\cdot)$ denotes the Lebesgue measure on the interval $[-1 / 2,1 / 2]$.

We now extend these results to the case where there is noiseless feedback from the receiver to the transmitter.

Theorem 3. Suppose that the fading $\left\{H_{k}\right\}$ and the specular component $d$ are such that $\left\{H_{k}-d\right\}$ is a stationary, ergodic, finite-energy, circularly-symmetric, Gaussian process. Then under a peak-power constraint noiseless feedback from the receiver to the transmitter does not increase the asymptotic channel capacity in the sense that,

$$
\lim _{\mathrm{SNR} \uparrow \infty} \frac{C_{\mathrm{FB}}^{\mathrm{PP}}(\mathrm{SNR})}{\log \mathrm{SNR}}=\lim _{\mathrm{SNR} \uparrow \infty} \frac{C^{\mathrm{PP}}(\mathrm{SNR})}{\log \mathrm{SNR}} .
$$

That is, the pre-log in presence of noiseless feedback $\Pi_{\mathrm{FB}}^{\mathrm{PP}}$ is given by (19).

\section{REFERENCES}

[1] Lapidoth, Amos and Moser, Stefan M., "Capacity bounds via duality with applications to multipleantenna systems on flat fading channels," IEEE Transactions on Information Theory, vol. 49, no. 10, pp. 2426-2467, October 2003.

[2] Lapidoth, Amos, "On the asymptotic capacity of fading channels," Submitted, available at $<$ http: //www. i si.ee.ethz.ch/ lapidoth>, 2003.
[3] Lapidoth, Amos, "On the high SNR capacity of stationary Gaussian fading channels," in Proceedings FortyFirst Allerton Conference on Communication, Control and Computing, Allerton House, Monticello, Illinois, October 1-3, 2003.

[4] Koch, Tobias, "On the asymptotic capacity of multipleinput single-output fading channels with memory," M.S. thesis, Signal and Information Processing Laboratory, ETH Zurich, Switzerland, April 2004, supervised by Prof. Dr. Amos Lapidoth. 